\title{
Do pregnant women living in endemic regions of chronic kidney disease of unknown origin (CKDu) show pathological changes in the placenta?
}

\author{
S A Karunananda ${ }^{a}$, R Waduge ${ }^{b}$
}

\begin{abstract}
There had not been much research to find possible involvement of other organs other than the kidney in CKDu. The effects on pregnancy due to renal impairment in general is known ${ }^{2,3}$ but what other damage could be inflicted by the unknown aetiology of CKDu on feto placental unit remains unknown. This injury could precede overt renal impairment. One such vulnerable organ is the placenta as both kidneys and placenta share similar function as filtration barriers. It is logical to assume if an agent could affect the interstitium of the kidney, similar adverse effects could be imposed on the interstitium of haemochorionic human placenta. This study was designed with the objective of analysing possible effects on human placental structure of patients who have been exposed to the probable aetiological factor responsible for CKDu. Obviously, anything affecting placenta would adversely affect fetus, its growth and organ development. If the agent passed through to the fetus, there could be similar direct damage to fetal kidneys too.

240 placental samples of women who had lived at least 10 years in a highly endemic area were compared with a similar number from those who were not so exposed. All samples were processed at the department of pathology and reported on by the second author. There was statistically insufficient evidence to conclude that population proportions of two categories were significantly different. The differences between categories and abnormalities were statistically insignificant. Since there was no noticeable Light Microscopic changes observed attributable to an unknown pathological agent, it was concluded that during a 9-months period of exposure of the placenta, no significant changes occurred even in the most endemic areas. This data was useful as women attending antenatal clinics could be reassured that no ill effect of the agent is known and it would be unnecessary to abandon home during pregnancy for safety of the fetus.
\end{abstract}

Key words: CKDu, Sri Lanka, pregnancy, placenta

Sri Lanka Journal of Obstetrics and Gynaecology 2021; 43: 31-38

DOI: http://doi.org/10.4038/sljog.v43i1.7977

a Senior Lecturer in Obstetrics and Gynaecology, Faculty of Medicine, University of Peradeniya, Sri Lanka.

b Senior Lecturer, Department of Pathology, Faculty of Medicine, University of Peradeniya, Sri Lanka.

Correspondence: SAK, e-mail: asokakk@hotmail.com

(iD) https://orcid.org/0000-0001-8811-0148

Received $28^{\text {th }}$ February 2021

Accepted after revision 22 ${ }^{\text {nd }}$ March 2021 


\section{Introduction}

Chronic Kidney Disease of unknown aetiology (CKDu) is a new and predominant form of CKD, which is endemic among several rural farming communities in and around the North Central, Uva and North Western Provinces of Sri Lanka, including Dehiattakandiya, Girandurukotte, Kabithigollawa, Medawachchiya, Medirigiriya, Nikawewa and Padaviya. Siripura, Horowpathana, Wilgamuwa.

It has become a priority issue in health system due to the dramatic increase in its prevalence and the severity of the outcome having reached epidemic proportions with ever increasing numbers of patients and deaths; (approximately 1400 deaths annually occur nationwide.)

Apart from Sri Lanka, other geographic "hot spots" of CKDu are El Salvador, Guatemala, Mexico, Nicaragua, Bulgaria, Croatia, Serbia and India. First evidence of CKDu was in 1950 Europe-Balkan region; In Sri Lanka it was 2000. It has been recognized as a global population health issue due to its mysterious nature.

The unique feature of the disease is that, it has no association with the well-known risk factors of renal injury such as diabetes, hypertension or chronic glomerulonephritis. The onset of the disease appears to be asymptomatic, and by the time patients seek treatment, the kidneys have reached a stage of irreversible damage (End Stage Renal Disease [ESRD]). It is logical to assume that a large proportion of pregnant women from the endemic areas are thus exposed or even diseased but yet undiagnosed due to this silent nature of the initial stages.

The disease appears to affect mainly the proximal tubules and the interstitium giving rise to characteristic, recognizable histopathological and clinical features. Clinically, the disease is characterized by tubular proteinuria, usually $\beta 2$-microglobulinuria. The histological appearance of the disease reveals a tubular-interstitial pathology that can commonly be observed in toxic nephropathies ${ }^{3}$. To date, there is no unequivocally acceptable evidence to recognize the possible environmental causative factors that is responsible for the disease.

The Health Ministry circular criteria are used to diagnose CKDu in current Sri Lankan clinical practice. It defines the diagnostic criteria for CKDu as Chronic
Kidney Disease in patients in the absence of past history or while on current treatment of diabetes mellitus and chronic and/or severe hypertension, snake bites, urological disease of known aetiology and glomerulonephritis. Patients should also have a normal HbA1C2 (Glycated Haemoglobin) < 6.5\% and Blood Pressure at $<160 / 100 \mathrm{~mm} \mathrm{Hg}$ or $140 / 90 \mathrm{~mm} \mathrm{Hg}$ on up to two antihypertensive agents.

CKDu patients are relatively younger than patients with CKD due to hypertension and diabetes making women in childbearing age vulnerable. Mean age of the population was 47.8 years (SD+/-13.7).

A case study has found, together with anecdotal evidence, that the most vulnerable group are men over 40 years of age, who have been working as rice farmers for over a decade. Though the age-standardised prevalence of Stage 3 and 4 of CKDu is higher in males than female, in, it is commoner amongst females in stages 1 and 2.

Although there are several on-going research projects to ascertain the aetiology, the definitive cause remains unclear todate. Furthermore there had not been much research to find any possible involvement of other organs due to this unknown aetiology causing CKD. One of the organs which could be affected is the placenta. It has been known that both organs share similar histological ultrastructure which is evidenced by occurrence of albuminuria in pre-eclampsia. It is logical to assume if an agent could affect the interstitium of the kidney, similar adverse effect could be affected on the interstitium of the haemochorionic human placenta.

The aim of this research was to find out whether any effect is exerted on the placental ultrastructure of women in areas where CKDu is most prevalent. If it was found to be so;

A follow up study was to be conducted to look for any fetal effects like, higher incidence of congenital malformations, intrauterine growth restriction or intrauterine death.

\section{Objectives}

To analyse the possible effects of the unidentified agent causing CKDu in selected endemic areas on human placental microstructure. There is no objective in trying to ascertain what the agent. 


\section{Materials and methods}

A descriptive cross sectional study was carried-out with 420 placental samples, divided into two similar categories as Exposed and Controls. The study sample was selected as pregnant mothers admitted to the antenatal wards for their confinement to 12 selected hospitals; namely, Teaching Hospital Peradeniya (controls only), District General Hospital Polonnaruwa, Anuradhapura and Kurunegala, Base Hospitals
Mahiyanganaya, Medirigiriya, Kebithigollewa and Dambulla, District Base Hospital Dehiattakandiya, District Hospitals Medawachchiya and Girandurukotte, Government Rural Hospital Nikawewa, as these are pre identified as high incidence pockets according to current geographical distribution of CKDu in Sri Lanka.

These sampling areas are demarcated in the map in relation to endemic zones.

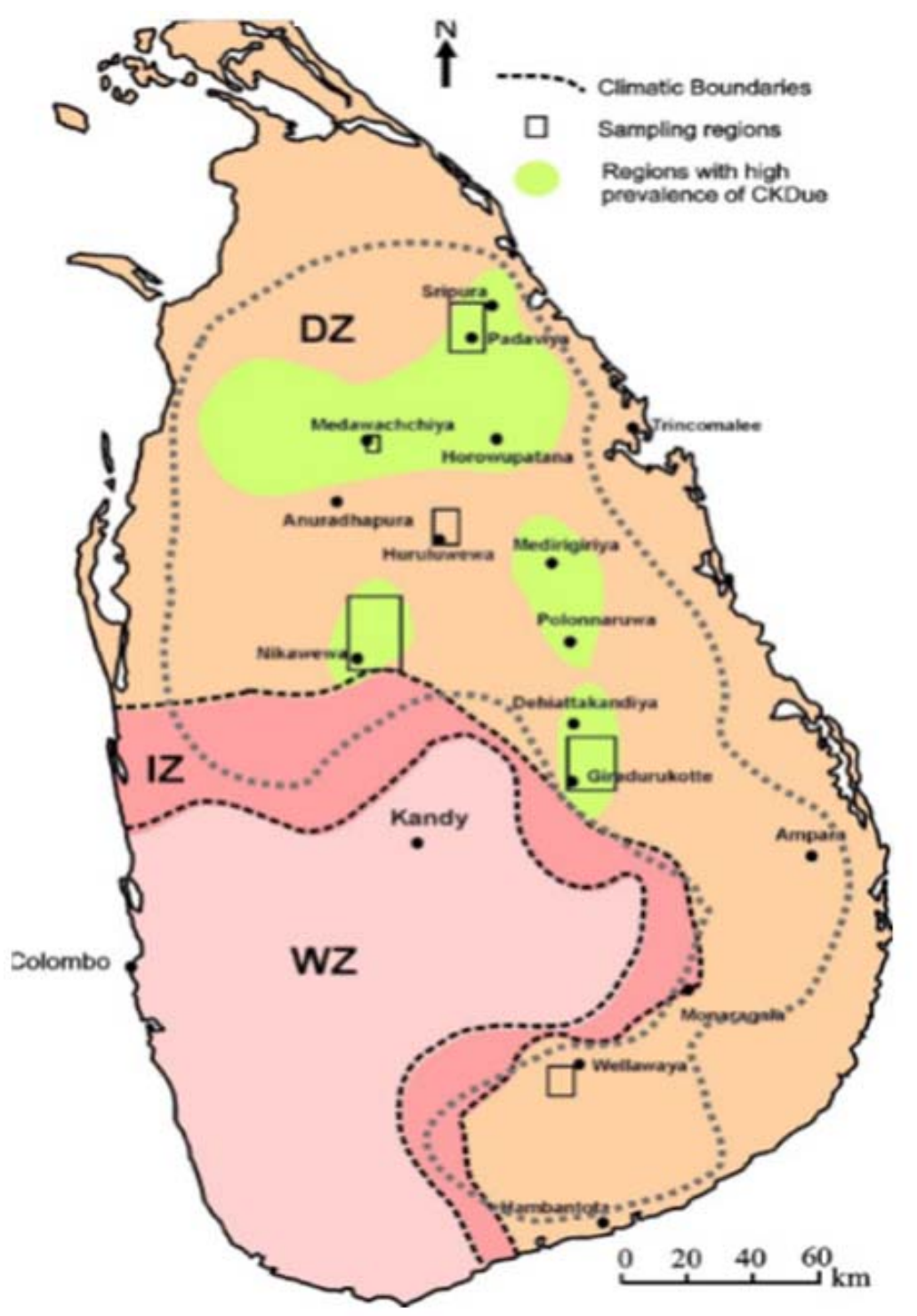

Map of sampling areas in relation to endemicity. 
The average period of residency in an endemic area of CKDu was selected as the study variable.

A pregnant mother whose duration of residency in an endemic area of CKDu exceeding 10 years was considered as an Exposed person. Pregnant women whose duration of residency in an endemic area of CKDu of less than 10 years in an endemic areas or those who lived in a non-endemic area of CKDu were considered as Controls. No randomization techniques were used.

Two hundred and ten pregnant mothers admitted for confinement from endemic areas and two hundred and ten from non-endemic areas for CKDu were selected according to the inclusion and exclusion criteria, so that 420 placental samples were collected in total. Maternal age was not used to stratify data analysis as it was not felt to influence possible injury from an exogenous source over and above the duration of exposure.

Inclusion criteria were having lived more than 10 years in endemic or non-endemic areas of CKDu; and having lived less than 10 years in endemic area non consenting, severely ill patients or those with pre identified placental pathologies were excluded. Subjects with confounding factors: smoking, alcohol, high BMI, anaemia, diabetes, hypertension, connective tissue disorders, previous history of snake bites, leptospirosis, malaria, or thalassemia was also not included. Patients with overt renal disease were similarly excluded as the objective was not to confound the effects of renal failure on pregnancy but only that of any aetiological agent acting directly on the placental unit.

Written permission was obtained from DDG-Ministry of Health, Provincial and Regional Directors of Health Services and Directors of selected hospitals. Nursing Officers and Family Health Officers of Labour rooms who voluntarily participated, were assigned to obtain the informed written consent from the patients and obtain basic details. These officers were selected after getting consent to ensure their voluntary participation. Basic details of patients included name, age and address and average period of residency in the area, family history of CKDu for the proper identification purpose; all subjects underwent the dipstick method to analyze urinary protein.
After delivery, relevant samples of the placenta $23 \mathrm{~cm}$ sections including membranes and a 0.5 " cross section sample from umbilical cord were obtained within two hours, by trained Nursing officers or Midwives and the rest of the placental tissue was sent for routine disposal. Placental samples were fixed in formalin.

The samples were collected by authorized persons from Faculty of Medicine, University of Peradeniya visiting each hospital and these samples were dispatched to the Department of Pathology, Faculty of Medicine, and University of Peradeniya. Specimens were mounted on slides and histopathological analysis of the samples was carried out to identify any abnormalities in placentas; inflammation, fibrosis, vasculitis, calcification, syncitial-knots and reduction of villi.

Final reports obtained after this analysis were fed in Microsoft Excel 2013 and these collected data was summarized and analyzed using SPSS version 20. Relative prevalence was calculated. Chi-Square test and Z-test were applied for categorical variables with 95\% Confidence.

\section{Results}

The compositions of the two groups were broadly similar in age composition parity and socioeconomic strata. The ethnicity with some bearing on diet and food preparation habits (vegetarian or not) was also not significantly dissimilar.

Out of entire population, $86.67 \%$ was with no inflammation with similar prevalence for both categories. prevalence of fibrosis was $0.24 \%$ with exposed. vasculitis was not identified in entire population. Prevalence of calcification was $10.95 \%$ and $12.38 \%$ for Controls and Exposed respectively. Syncytial-knots were identified only in Controls as $0.48 \%$. Prevalence of reduction of villi was $1.19 \%$ and $3.33 \%$ for Controls and Exposed respectively. There was statistically insufficient evidence to conclude that population proportions of two categories were significantly different. The differences between categories and abnormalities were statistically insignificant. Since there was statistically insignificant association between category and each abnormality, and also with the severity of each abnormality, the two categories can be considered to be almost similar. 


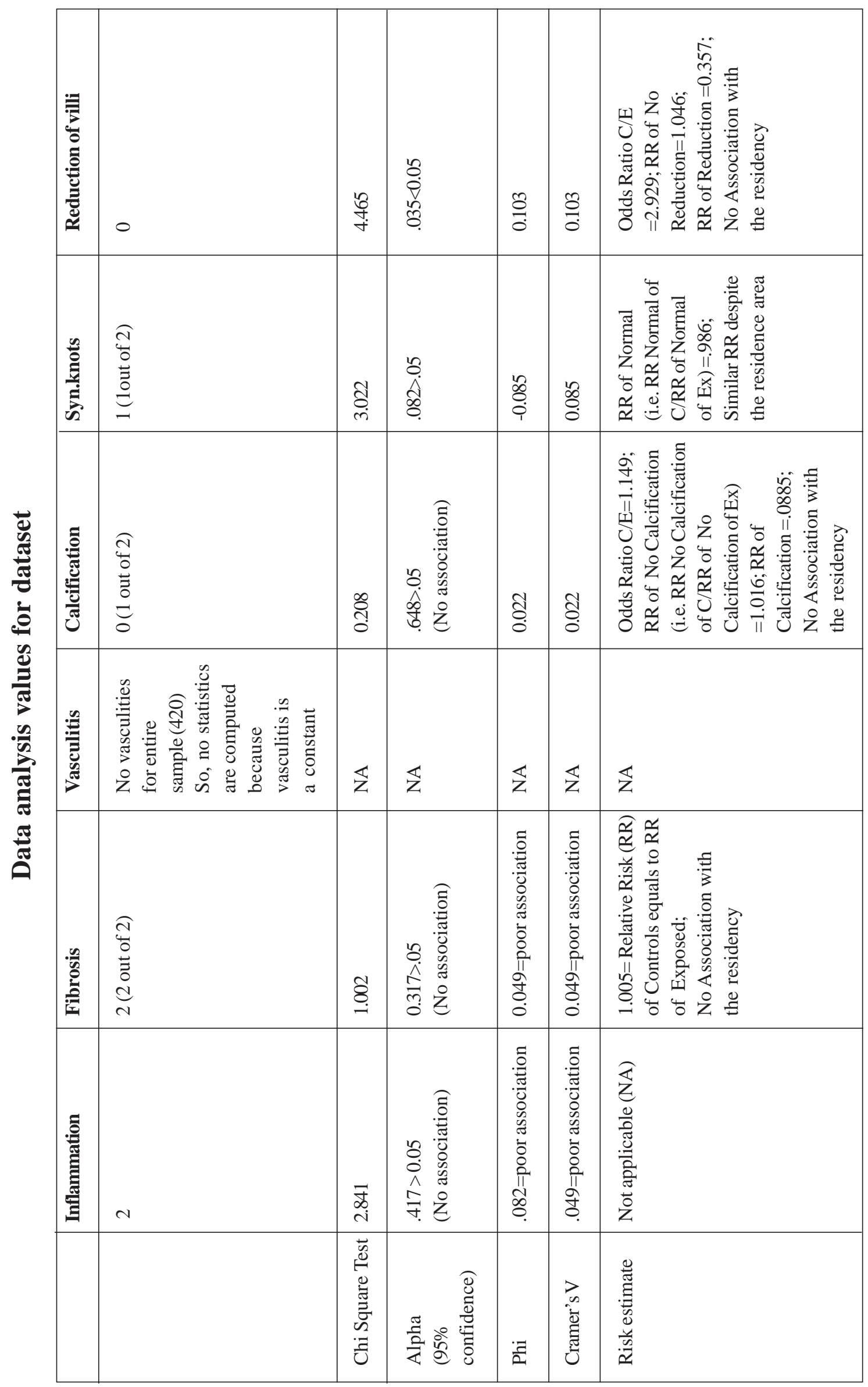




\begin{tabular}{|c|c|c|c|c|c|c|c|c|c|c|c|c|}
\hline 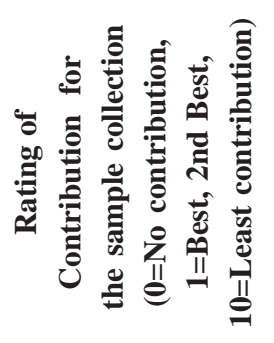 & $N$ & - & $\wedge$ & 0 & $m$ & n & $\sigma$ & & & & † $\infty$ & \\
\hline 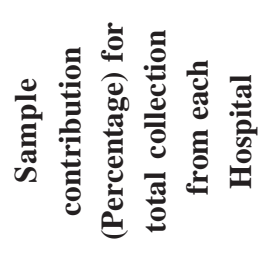 & $\begin{array}{l}M^{m} \\
\stackrel{\infty}{\sim}\end{array}$ & 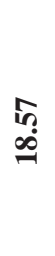 & $\stackrel{\vec{m}}{\vec{m}}$ & $\stackrel{8}{\circ}$ & 勇 & $\begin{array}{l}\text { L } \\
\dot{+} \\
-\end{array}$ & 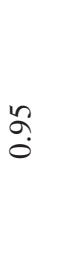 & $\stackrel{8}{0}$ & 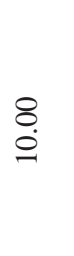 & 吕 & ن. & \\
\hline 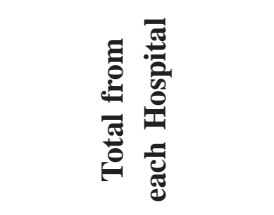 & $\hat{\Sigma}$ & $\stackrel{\infty}{\Re}$ & 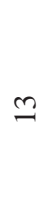 & 0 & 8 & 욤 & $\nabla$ & 0 & $\mathscr{7}$ & $\sim$ & 8 足 & ฟิ \\
\hline 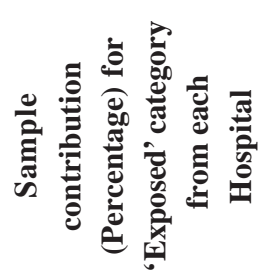 & $\stackrel{8}{\circ}$ & $\begin{array}{l}\vec{\infty} \\
\vec{j}\end{array}$ & $\begin{array}{l}\stackrel{゚}{\leftarrow} \\
\stackrel{+}{*}\end{array}$ & : & స్. & $\begin{array}{l}\stackrel{D}{~} \\
\stackrel{+}{N}\end{array}$ & $\stackrel{\text { If }}{\stackrel{-}{-}}$ & $\stackrel{8}{0}$ & $\begin{array}{c}\vec{\infty} \\
\dot{\infty}\end{array}$ & 哭 & 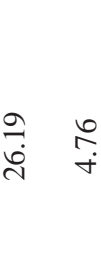 & \\
\hline 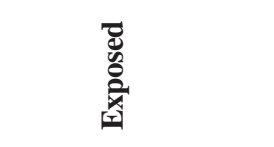 & 0 & in & $\stackrel{9}{9}$ & $\circ$ & 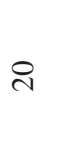 & in & m & 0 & $\infty$ & $\sim$ & 另 9 & $\stackrel{\text { ㄱ }}{2}$ \\
\hline 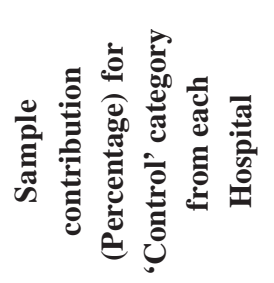 & 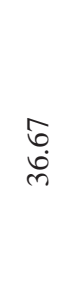 & ભm & $\stackrel{\text { q }}{\mathrm{I}}$ & $\stackrel{0}{8}$ & ભm & $\stackrel{m}{m}$ & $\stackrel{\infty}{0}$ & $\stackrel{8}{\circ}$ & تُ & $\stackrel{8}{\circ}$ & $\begin{array}{lll}\stackrel{\infty}{\rightarrow} & \stackrel{9}{+} \\
\end{array}$ & \\
\hline 意 & $\hat{\Sigma}$ & $\stackrel{\sim}{\infty}$ & $m$ & 0 & g & n & $\neg$ & 0 & $\vec{m}$ & 0 & 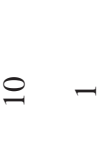 & 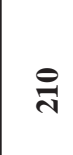 \\
\hline 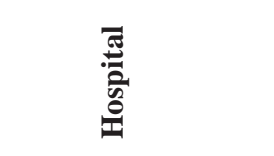 & 产 & $\stackrel{T}{\Sigma}$ & 띰 & ن & $\Sigma$ & 茛 & $\sum$ & $\underline{v}$ & 畄 & 兑 & $\circ \quad$ & 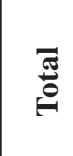 \\
\hline
\end{tabular}




\section{Discussion}

The anxiety caused to pregnant women from endemic areas of CKDu would be mainly related to risk of fetal involvement. It would be simple logic to assume by pregnant women that whatever damages their kidneys could filter through and damage baby's kidneys leaving alone more advanced knowledge needed to be concerned regarding possibility of placental damage. The near absolute absence of data related specifically to $\mathrm{CKDu}$ in pregnancy puts the counselling medical personnel in a difficult situation as was experienced once by the author prompting the study. It is not possible to reassure sans factual evidence. There was no material on at least the internet to refer to either. Paucity of data is such that there is no similar series to even compare findings of this study. This is based on a main health priority at the time of writing. All available studies on CKDu in Sri Lanka at the time of writing were focussed on the aetiology or prevalence $e^{7,8,9,10}$.

Logically, it was expected to observe changes to interstitium of placenta similar to the interstitium of kidney caused by CKDu. Since there was no noticeable light microscopic changes observed, it can be concluded that during a 9 months period of exposure of the placenta, no significant changes were observable even in the most endemic areas. The placenta actually exists for 8 out of 9 months of pregnancy even if it went up to term. These findings would provide the much needed basis for any reassurances given to women of endemic areas of CKDu.

\section{Conclusions}

Since severe stages of placental abnormalities were not revealed and there is no statistically significant difference amongst placentae of controls and exposed pregnancies, it can be concluded that the duration of pregnancy of up to 9 months may not be sufficiently long enough to create significant changes to the interstitium of placenta by the aetiological factors of $\mathrm{CKDu}$. One possible reason maybe that the kidney is more vulnerable due to its size and the high filtration rate in comparison to the placenta. It is also possible that light microscopy not being revealing enough in early stages of involvement calling for electron microscopic scrutiny. There should therefore be no undue case for alarming pregnant women about possible damage to at least the placenta and most likely the fetus. A larger scale study on renal functions of new-borns of endemic areas is still in order as it is possible for $\mathrm{CKDu}$ agent to simply filter through the placenta without affecting it, but inflict damage on fetal kidneys.

\section{Author declarations}

Authors' contributions: Initial concept planning and by main author Dr S A Karunananda. All data processing and processing of 420 histological samples by $\mathrm{Dr} R$ Waduge.

Manuscript drafting and initial editing was by both authors. Data and sample collection performed by technical staff of the Department and Research Assistants, Midwives coordinated by Kumudumali Perera, Research Assistant.

Conflicts of interest: There are no conflicts of interest or any financial gains of any personal nature. The data is freely available at Department of Pathology regarding processed specimens.

Ethical approval: Ethical clearance obtained from Ethics and Research Committee, Faculty of Medicine, University of Peradeniya.

Financial assisstance: Research grant of Rs 400,000 received from the University of Peradeniya.

Informed consent: The study in preliminary form was presented as an abstract at SLCOG sessions in August 2019. This is the final completed paper.

\section{Acknowledgements}

The assistance provided by Provincial Directors of Health and Hospital staff in areas concerned: Kumudumali Perera, Technical Officers, Department of Pathology, University of Peradeniya are acknowledged with thanks.

\section{References}

1. Edipidis K. Hippokratia Pregnancy in women with renal disease. Yes or no?. 2011; 15(Suppl 1): 8-12.

2. Ramin SM, Vidaeff AC, Yeomans ER, Gilstrap LC. Chronic renal disease in pregnancy, Obstet Gynecol. 2006; 108(6): 1531-9.

3. Bili E, Tsolakidis D, Stangou S, Hippokratia. Pregnancy management and outcome in women with chronic kidney disease. Hippokratia Quarterly Medical Journal 2013; 17(2): 163-8. 
4. Noble A, Amerasinghe P, Manthrithilake H. Review of Literature on Chronic Kidney Disease of Unknown Etiology (CKDu) in Sri Lanka.

5. Ramin SM, Vidaeff AC, Yeomans ER, Chronic renal disease in pregnancy, Obstet Gynecol. 2006; 108(6): 1531-9.

6. Surian M, Imbasciati E, Cosci P Nephron. A study of 123 pregnancies in patients with primary and secondary glomerular diseases. Glomerular Disease and Pregnancy. 1984; 36(2): 101-5.

7. Athuraliya T, Abeysekera D, Amerasinghe PH, Kumarasiri P, Dissanayake V. Prevalence of chronic kidney disease in two tertiary care hospitals: high proportion of cases with uncertain aetiology. Ceylon Med J. 2009; 54(1): 23-25.
8. Jayatilake N, Mendis S, Maheepala P, Mehta FR. Chronic kidney disease of uncertain aetiology: prevalence and causative factors in a developing country. BMC Nephrol. 2013; 14(1): 180.

9. Jayasekara KB, Dissanayake DM, Sivakanesan R, Ranasinghe A, Karunarathna RH, Priyantha Kumara GW. Epidemiology of chronic kidney disease, with special emphasis on chronic kidney disease of uncertain aetiology, in the north central region of Sri Lanka. J Epidemiol. 2015; 25(4): 275-80.

10. Wanigasuriya KP, Peiris-John RJ, Wickremasinghe R, Hittarage A. Chronic renal failure in North Central Province of Sri Lanka: an environmentally induced disease. Trans R Soc Trop Med Hyg. 2007; 101(10): 1013-7. 